\title{
Infinite dimensional Cauchy-Kowalevski and Holmgren type theorems
}

\author{
Jiayang $\mathrm{YU}^{1} \& \mathrm{Xu}$ ZHANG ${ }^{2, *}$ \\ ${ }^{1}$ School of Mathematics, Sichuan University, Chengdu 610064, P. R. China; \\ 2 School of Mathematics, Sichuan University, Chengdu 610064, P. R. China \\ Email: jiayangyu@scu.edu.cn, zhang_xu@scu.edu.cn
}

\begin{abstract}
The aim of this paper is to show Cauchy-Kowalevski and Holmgren type theorems with infinite number of variables. We adopt von Koch and Hilbert's definition of analyticity of functions as monomial expansions. Our Cauchy-Kowalevski type theorem is derived by modifying the classical method of majorants. Based on this result, by employing some tools from abstract Wiener spaces, we establish our Holmgren type theorem.
\end{abstract}

Keywords Cauchy-Kowalevski theorem, Holmgren theorem, monomial expansions, abstract Wiener space, divergence theorem, method of majorants

MSC(2010) Primary 35A10, 26E15, 46G20; Secondary 46G20, 46G05, 58B99.

Citation: Jiayang Yu, Xu Zhang. Infinite dimensional Cauchy-Kowalevski and Holmgren type theorems. Sci China Math, 2019, 60, doi:

\section{Introduction}

The classical Cauchy-Kowalevski theorem asserts the local existence and uniqueness of analytic solutions to quite general partial differential equations with analytic coefficients and initial data in the finite dimensional Euclidean space $\mathbb{R}^{n}$ (for any given $n \in \mathbb{N}$ ). In 1842, A. L. Cauchy first proved this theorem for the second order case; while in 1875, S. Kowalevski proved the general result. Both of them used the method of majorants. Because of its fundamental importance, there exists continued interest to generalize and/or improve this theorem (See $[2,10,24,26-29,31-33,35]$ and the references cited therin). In particular, some mathematicians studied abstract forms of Cauchy-Kowalevski theorem in the context of Banach spaces and Fréchet derivatives. For example, the linear cases were considered by L. Ovsjannikov ([29]) and F. Trèves ([32]). Later, L. Nirenberg ([27]) obtained a nonlinear form, while T. Nishida ([28]) simplified Nirenberg's proof and obtained a more general version, M. Safonov ([31]) gave another proof of Nishida's theorem. There are also some abstract Cauchy-Kowalevski theorems in this respect $([2,26,33])$.

On the other hand, Holmgren's uniqueness theorem states the uniqueness of solutions to linear partial differential equations with analytic coefficients in much larger class of functions than analytic ones. In 1901, E. Holmgren ([18]) first proved this theorem for the case of $n=2$ in the context of classic solutions, while in 1949, F. John ([21]) extended it to the general case of $n$ variables. Later the result was extended

\footnotetext{
* Corresponding author
} 
first to the setting of distribution solutions by L. Hörmander $([19,20])$ and then to that of hyperfunction solutions by H. Hedenmalm ([16]). All of the above works are addressed to the case of finite dimensional spaces. In the 1970s, B. Lascar $([23,24])$ gave a Banach space version of Holmgren's uniqueness theorem. Unfortunately, the proof of Lascar's result in this respect is incomplete and questionable, and therefore, about thirty years later, in [2] M. Chaperon said that no infinite-dimensional version of Holmgren's theorem seems to be known.

The main purpose of this paper is to establish Cauchy-Kowalevski and Holmgren type theorems on $\mathbb{R}^{\infty}$, which is a countable Cartesian product of $\mathbb{R}$. In some sense, this is quite natural because $\mathbb{R}^{\infty}$ is an infinite dimensional counterpart of $\mathbb{R}^{n}$, the $n$-fold Cartesian product of $\mathbb{R}$. Since there are various different topologies on $\mathbb{R}^{\infty}$, we have more freedom and flexibility to introduce suitable assumptions on equations under consideration. Nevertheless, a large family of local derivatives of functions on $\mathbb{R}^{\infty}$ make the analysis in our work more complicated. Clearly, we cannot apply the known abstract Cauchy-Kowalevski and Holmgren type theorems (in the literatures) in the setting of Banach spaces to obtain our results. Indeed, our working space, $\mathbb{R}^{\infty}$, is NOT a Banach space!

There exists many different definitions of analyticity in infinite dimensions. As far as we know, the concept of analyticity for functions of infinitely many variables began with H. von Koch in 1899 ([22]). H. von Koch ([22]) introduced a monomial approach to holomorphic functions on infinite dimensional polydiscs which was further developed by D. Hilbert in 1909 ([17]). After the pioneering work of H. von Koch and D. Hilbert, it is clear from M. Fréchet [8,9] and R. Gâteaux [11,12] that the power series expansion in terms of homogeneous polynomials seems more suitable for the analyticity in the context of Banach space (e.g., [5] for the extensive works on infinite dimensional complex analysis starting from 1960s). Nevertheless, in the recent decades, it was found that Hilbert's definition of analyticity is also useful in some problems. For example, in 1987, R. Ryan ([30]) discovered that every entire function $f$ on $\ell^{1}$ (the usual Banach space of absolutely summable sequences of real numbers) has a monomial expansion which converges and coincides with $f$; in 1999, L. Lempert ([25]) proved this holds for any open ball of $\ell^{1}$; while in 2009, A. Defant, M. Maestre and C. Prengel ([4]) showed that the monomial expansion of any holomorphic function on the Reinhardt domain $\mathcal{R}$ in a Banach sequence space converges uniformly and absolutely on any compact subsets of $\mathcal{R}$. In this work, we shall use the definition of analyticity introduced by H. von Koch and D. Hilbert.

We shall modify the classical method of majorants to derive a Cauchy-Kowalevski type theorem in $\mathbb{R}^{\infty}$. Based on this result, we then employ some tools from abstract Wiener spaces (developed by L. Gross in [15]) and especially an infinite dimensional divergence theorem ([13]) to establish a Holmgren type theorem with infinite number of variables. For the later, the basic idea is more or less the same as that in finite dimensions but the technique details are much more complicated. Indeed, it is well-known that, compared with its finite dimensional counterpart, the analysis tools in infinite dimensions are much less developed.

The rest of this paper is organized as follows. Section 2 is of preliminary nature, in which we first introduce suitable topologies for $\mathbb{R}^{\infty}$ and for $\mathbb{R}^{\infty}$ plus an infinity point; then, we give the definition of analyticity for functions of infinitely many variables; also, we present a brief introduction to the theory of abstract Wiener space and a divergence theorem. In Section 3, we show a Cauchy-Kowalevski type theorem of infinitely many variables. Section 4 is devoted to establishing our Holmgren type theorem.

We refer to [34] for the details of proofs of the results announced in this paper and some other results in this context.

\section{Preliminaries}

\section{$2.1 \quad$ A family of topologies}

There exists only one useful topology on finite dimensional space $\mathbb{R}^{n}$. However, we need to use a family of topologies on $\mathbb{R}^{\infty}$. 
Denote by $\mathscr{B}_{\infty}$ the class of sets (in $\left.\mathbb{R}^{\infty}\right):\left(x_{i}\right)+B_{r}^{\infty} \triangleq\left\{\left(x_{i}+y_{i}\right):\left(y_{i}\right) \in B_{r}^{\infty}\right\}$, where $\left(x_{i}\right) \in \mathbb{R}^{\infty}$, $r \in(0,+\infty)$ and $B_{r}^{\infty} \triangleq\left\{\left(y_{i}\right) \in \mathbb{R}^{\infty}: \sup _{1 \leqslant i<\infty}\left|y_{i}\right|<r\right\}$. Then $\mathscr{B}_{\infty}$ is a base for a topological space, denoted by $\mathscr{T}_{\infty}$. For any $\left(x_{i}\right),\left(y_{i}\right) \in \mathbb{R}^{\infty}$, define $d_{\infty}\left(\left(x_{i}\right),\left(y_{i}\right)\right) \triangleq \min \left\{1, \sup _{1 \leqslant i<\infty}\left|x_{i}-y_{i}\right|\right\}$. Then, it is easy to show the following result:

Proposition 2.1. The following assertions hold:

(1) $\left(\mathbb{R}^{\infty}, \mathscr{T}_{\infty}\right)$ is not a topological vector space;

(2) $\left(\mathbb{R}^{\infty}, \mathscr{T}_{\infty}\right)$ is compatible with the metric $d_{\infty}$ and $\left(\mathbb{R}^{\infty}, d_{\infty}\right)$ is a nonseparable complete metric space.

For any $p \in[1, \infty)$ and $\left(x_{i}\right),\left(y_{i}\right) \in \mathbb{R}^{\infty}$, define $\mathrm{d}_{p}\left(\left(x_{i}\right),\left(y_{i}\right)\right) \triangleq \min \left\{1,\left(\sum_{i=1}^{\infty}\left|x_{i}-y_{i}\right|^{p}\right)^{\frac{1}{p}}\right\}$. Then, $\left(\mathbb{R}^{\infty}, d_{p}\right)$ is a complete metric space. Denote by $\mathscr{T}_{p}$ the topology induced by the metric $d_{p}$, and by $\mathscr{B}_{p}$ the class of sets: $\left(x_{i}\right)+B_{r}^{p}$, where $\left(x_{i}\right) \in \mathbb{R}^{\infty}, r \in(0,+\infty)$ and $B_{r}^{p} \triangleq\left\{\left(x_{i}\right) \in \mathbb{R}^{\infty}:\left(\sum_{i=1}^{\infty}\left|x_{i}\right|^{p}\right)^{\frac{1}{p}}<r\right\}$. Obviously, $\mathscr{B}_{p}$ is a base for the topology space $\mathscr{T}_{p}$. Also, denote by $\ell^{p}\left(\right.$ resp. $\left.\ell^{\infty}\right)$ the usual Banach space of sequences $\left(x_{i}\right) \in \mathbb{R}^{\infty}$ so that $\left(\sum_{i=1}^{\infty}\left|x_{i}\right|^{p}\right)^{\frac{1}{p}}<\infty$ (resp. $\left.\sup _{1 \leqslant i<\infty}\left|x_{i}\right|<\infty\right)$.

Likewise, for any $p \in(0,1)$ and $\left(x_{i}\right),\left(y_{i}\right) \in \mathbb{R}^{\infty}$, define $\mathrm{d}_{p}\left(\left(x_{i}\right),\left(y_{i}\right)\right) \triangleq \min \left\{1,\left(\sum_{i=1}^{\infty}\left|x_{i}-y_{i}\right|^{p}\right)\right\}$. Then $d_{p}$ is a metric on $\mathbb{R}^{\infty}$. Denote by $\mathscr{T}_{p}$ the topology induced by the metric $d_{p}$, and by $\mathscr{B}_{p}$ the class of sets: $\left(x_{i}\right)+B_{r}^{p}$, where $\left(x_{i}\right) \in \mathbb{R}^{\infty}, r \in(0,+\infty)$ and $B_{r}^{p} \triangleq\left\{\left(x_{i}\right) \in \mathbb{R}^{\infty}: \sum_{i=1}^{\infty}\left|x_{i}\right|^{p}<r\right\}$. Similarly to Proposition 2.1, $\left(\mathbb{R}^{\infty}, \mathscr{T}_{p}\right)$ and $\mathscr{B}_{p}$ is a base for the topology space $\mathscr{T}_{p}$ when $0<p<\infty$.

Denote by $\mathscr{T}$ the (usual) product topology on $\mathbb{R}^{\infty}$. Now we have defined a family of topologies on $\mathbb{R}^{\infty}$. The following result shows some relations between these topologies.

Proposition 2.2. The inclusion relations $\mathscr{T} \varsubsetneqq \mathscr{T}_{\infty} \varsubsetneqq \mathscr{T}_{q} \varsubsetneqq \mathscr{T}_{p}$ hold for any $0<p<q<\infty$.

Let $\widetilde{\mathbb{R}^{\infty}} \triangleq \mathbb{R}^{\infty} \sqcup\{\infty\}$, where $\infty$ is any fixed point not belonging in $\mathbb{R}^{\infty}$. We consider the following family of sets (in $\widetilde{\mathbb{R}^{\infty}}$ ): $\mathscr{B}^{p} \triangleq \mathscr{B}_{p} \bigcup\left\{\left\{\left(x_{n}\right) \in \mathbb{R}^{\infty}: x_{n} \neq 0\right.\right.$ for each $n \in \mathbb{N}$ and $\left.\left.\sum_{n=1}^{\infty} \frac{1}{\left|x_{n}\right|^{p}}<r\right\} \sqcup\{\infty\}: r>0\right\}$ for $0<p<\infty$, and $\mathscr{B}^{\infty} \triangleq \mathscr{B}_{\infty} \bigcup\left\{\left\{\left(x_{n}\right) \in \mathbb{R}^{\infty}: x_{n} \neq 0\right.\right.$ for each $n \in \mathbb{N}$ and $\sup _{1 \leqslant n<\infty} \frac{1}{\left|x_{n}\right|}<$ $r\} \sqcup\{\infty\}: r>0\}$. Then $\mathscr{B}^{p}$ is a base for a topological space on $\widetilde{\mathbb{R}^{\infty}}$ which we will denoted by $\mathcal{T}^{p}$ for $0<p \leqslant \infty$. It is easily seen that the subspace topology of $\left(\widetilde{\mathbb{R}^{\infty}}, \mathcal{T}^{p}\right)$ on $\mathbb{R}^{\infty}$ is $\mathscr{T}_{p}$ but $\mathcal{T}^{p}$ is not the usual one-point compactification of $\mathscr{T}_{p}$ (and therefore we use the notion $\widetilde{\mathbb{R}^{\infty}}$ rather than $\widehat{\mathbb{R}^{\infty}}$ for the usual one-point compactification).

\subsection{Analyticity for Functions of Infinitely Many Variables}

We denote by $\mathbb{N}^{(\mathbb{N})}$ the set of all finitely supported sequences of nonnegative integers. For $\mathbf{x}=\left(x_{i}\right) \in \mathbb{R}^{\infty}$, $\alpha=\left(\alpha_{i}\right) \in \mathbb{N}^{(\mathbb{N})}$ with $\alpha_{k}=0$ for $k \geqslant n+1$ and some $n \in \mathbb{N}$, write $\mathbf{x}^{\alpha} \triangleq x_{1}^{\alpha_{1}} x_{2}^{\alpha_{2}} \cdots x_{n}^{\alpha_{n}}$, which is called a monomial on $\mathbb{R}^{\infty}$. The following definition of analyticity is essentially from [22] and [17].

Definition 2.3. Suppose $f$ is a real-valued function defined on a subset $D$ of $\mathbb{R}^{\infty}$ and $\mathbf{x}_{0} \in D$. If for each $\alpha \in \mathbb{N}^{(\mathbb{N})}$, there exist a real number $c_{\alpha}$ (depending on $f$ and $x_{0}$ ) such that the series $\sum_{\alpha \in \mathbb{N}^{(\mathbb{N})}} c_{\alpha} \mathbf{x}^{\alpha}$ is absolutely convergent for some $\mathbf{x}=\left(x_{i}\right) \in \mathbb{R}^{\infty}$ with $x_{i} \neq 0$ for each $i \in \mathbb{N}$, and for each $\mathbf{h}=\left(h_{i}\right) \in \mathbb{R}^{\infty}$ with $\left|h_{i}\right| \leqslant\left|x_{i}\right|$ for all $i \in \mathbb{N}$, it holds that $\mathbf{x}_{0}+\mathbf{h} \in D$ and

$$
f\left(\mathbf{x}_{0}+\mathbf{h}\right)=\sum_{\alpha \in \mathbb{N}^{(N)}} c_{\alpha} \mathbf{h}^{\alpha},
$$

then $f$ is called analytic near $\mathbf{x}_{0}$ (with the monomial expansion (2.1)). In this case, we write $D_{f}^{\mathbf{x}_{0}} \triangleq\{\mathbf{h} \in$ $\left.\mathbb{R}^{\infty}: \sum_{\alpha \in \mathbb{N}^{(\mathbb{N})}}\left|c_{\alpha} \mathbf{h}^{\alpha}\right|<\infty\right\}$, and call the set $D_{f}^{\mathbf{x}_{0}}$ the convergence domain of monomial expansion (2.1).

Definition 2.4. Suppose $f$ is a real-valued function defined on a subset $D$ of $\mathbb{R}^{\infty}, \mathbf{x}_{0} \in D$ and $0<p<\infty$. If there exists $\mathbf{x}=\left(x_{i}\right) \in \mathbb{R}^{\infty}$ with $\sum_{i=1}^{\infty} \frac{1}{x_{i} p^{p}}<\infty$ such that $f$ is analytic near $\mathbf{x}_{0}$ with the monomial expansion (2.1) and $\sum_{\alpha \in \mathbb{N}^{(\mathbb{N})}}\left|c_{\alpha} \mathbf{x}^{\alpha}\right|<\infty$, then the monomial expansion of $f$ near $\mathbf{x}_{0}$ is called absolutely convergent at a point near $\infty$ in the topology $\mathcal{T}^{p}$.

Example 2.5. (Riemann Zeta Function) Recall that the Riemann zeta function is defined by $\zeta(s)=\sum_{n=1}^{\infty} \frac{1}{n^{s}}$ when $s \in \mathbb{C}$ with $\operatorname{Re}(s)>2$. Suppose that $\left\{p_{n}\right\}_{n=1}^{\infty}$ is the collection of all positive prime numbers. Then for $s \in \mathbb{R}$ with $s>2$ we have $\zeta(s)=\prod_{n=1}^{\infty} \frac{1}{1-p_{n}^{-s}}=\sum_{\alpha=\left(\alpha_{1}, \cdots, \alpha_{n}\right) \in \mathbb{N}^{(\mathbb{N})}} p_{1}^{-s \alpha_{1}} \cdots p_{n}^{-s \alpha_{n}}=$ 
$\sum_{\alpha \in \mathbb{N}^{(\mathbb{N})}}\left(p_{1}^{-s}, \cdots, p_{n}^{-s}, \cdots\right)^{\alpha}=f\left(p_{1}^{-s}, \cdots, p_{n}^{-s}, \cdots\right)$, where $f(\mathbf{x}) \triangleq \sum_{\alpha \in \mathbb{N}^{(\mathbb{N})}} \mathbf{x}^{\alpha}$ with definition domain $D=\left\{\mathbf{x}=\left(x_{i}\right) \in \mathbb{R}^{\infty}: \sum_{i=1}^{\infty}\left|x_{i}\right|<1\right\}$ is the function of geometric series of infinitely many variables.

Naturally, the notion of majority function is as follows:

Definition 2.6. Suppose $f$ and $F$ are analytic near $\mathbf{x}_{0} \in \mathbb{R}^{\infty}$ with monomial expansions $f(\mathbf{x})=$ $\sum_{\alpha \in \mathbb{N}^{(\mathbb{N})}} c_{\alpha}\left(\mathbf{x}-\mathbf{x}_{0}\right)^{\alpha}$ and $F(\mathbf{x})=\sum_{\alpha \in \mathbb{N}^{(\mathbb{N})}} C_{\alpha}\left(\mathbf{x}-\mathbf{x}_{0}\right)^{\alpha}$, respectively, where $c_{\alpha} \in \mathbb{R}$ and $C_{\alpha} \geqslant 0$ for each $\alpha \in \mathbb{N}^{(\mathbb{N})}$. If $\left|c_{\alpha}\right| \leqslant C_{\alpha}$ for all $\alpha \in \mathbb{N}^{(\mathbb{N})}$, then $F$ is called a majority function of $f$ near $\mathbf{x}_{0}$.

Suppose $B$ is a real Banach space and $U$ is an open set of $B$. For each $n \in \mathbb{N}$, a function $g$ from $B$ into $\mathbb{R}$ is called continuous $n$-homogeneous polynomial if there exists a continuous $n$-linear map $T$ from $\prod_{i=1}^{n} B$ into $\mathbb{R}$ such that $g(x)=T(x, \cdots, x)$ for each $x \in B$. For $n=0$, we call any function from $B$ into $\mathbb{R}$ with constant value a continuous 0-homogeneous polynomial. A function $f$ (defined on a subset $D$ of $B$ ) is called analytic on some subset $U \subset D$ if for each $\xi \in U$ there exist a sequence $\left\{P_{n} f(\xi)\right\}_{n=0}^{\infty}$ of continuous $n$-homogeneous polynomials on $B$ and a radius $r>0$ such that $\xi+B(r) \subset U$ and $f(\xi+x)=\sum_{n=0}^{\infty} P_{n} f(\xi)(x)$ uniformly in $B(r)$, where $B(r) \triangleq\{y \in B:\|y\|<r\}$. It is easy to see that, the function $g(\cdot) \triangleq f(\xi+\cdot)$ is Fréchet differentiable in $B(r)$ (See [5] for more details).

We emphasize that $\mathbb{R}^{\infty}$ is not a norm space. Hence, the analyticities by monomial expansions and by power series expansions are two distinct notions. Nevertheless, for every $\mathbf{x} \in \mathbb{R}^{\infty}, r \in(0,1)$ and $p \in[1,+\infty]$, it holds that $\mathbf{x}+B_{r}^{p} \subset \mathbb{R}^{\infty}$. Motivated by this simple observation, we have the following definition.

Definition 2.7. Suppose $p \in[1, \infty], U \subset \mathbb{R}^{\infty}, f$ is a real-valued function defined on $U$ and $\mathbf{x} \in U$. We call $f$ is Fréchet differentiable with respect to $\ell^{p}$ in a neighborhood of $\mathbf{x}$ in the topology $\mathscr{T}_{p}$ if there exists $r \in(0,1)$ such that $\mathbf{x}+B_{r}^{p} \subset U$, and the function defined by $g(\cdot) \triangleq f(\mathbf{x}+\cdot)$ is Fréchet differentiable with respect to the Banach space $\ell^{p}$ in $B_{r}^{p}$.

\subsection{Abstract Wiener Space and Derivatives}

Let us recall the notion of abstract Wiener space, which will be of crucial importance in the proof of the Holmgren type theorem. The materials in this subsection are from $[6,7,14,15]$.

Suppose $X$ is a real separable Banach space, and denote by $X^{*}$ its dual space and by $\langle\cdot, \cdot\rangle$ the natural pairing from $X^{*} \times X$ into $\mathbb{R}$. Let $Y$ be another Banach space. Denote by $\mathcal{L}(X ; Y)$ the Banach space of all bounded linear operators from $X$ to $Y$, with the usual operator norm (When $Y=X$, we simply write $\mathcal{L}(X)$ instead of $\mathcal{L}(X ; Y))$. A subset $C$ of $X$ is called a cylinder set if it is of the form:

$$
C=\left\{x \in X:\left(\left\langle y_{1}, x\right\rangle, \cdots,\left\langle y_{n}, x\right\rangle\right) \in E\right\},
$$

where $n \in \mathbb{N}, y_{1}, \cdots, y_{n} \in X^{*}$ and $E$ is a Borel set in $\mathbb{R}^{n}$. If $L$ is a finite-dimensional subspace of $X^{*}$ such that $\left\{y_{1}, \cdots, y_{n}\right\} \subset L$, then $C$ is said to be based on $L$. Clearly, the collection of cylinder sets in $X$ is an algebra $\mathscr{R}$ and the collection of cylinder sets based on $L$ is a $\sigma$-algebra which will be denoted by $\mathscr{S}_{L}$. We call a nonnegative set function $\mu$ on $\mathscr{R}$ a cylinder set measure on $X$ if $\mu(X)=1$ and $\mu$ is countably additive on $\mathscr{S}_{L}$ for each finite-dimensional subspace $L$ of $X^{*}$.

Suppose $H$ is a real separable Hilbert space with inner product $(\cdot, \cdot)$ and norm $|\cdot|=\sqrt{(\cdot, \cdot)}$. Then every cylinder set of $H$ is of the form

$$
C=\{x \in H: P x \in E\},
$$

where $P$ is a finite-dimensional projection in $H$ and $E$ is a Borel set in $P H$. For any $t>0$, a (typical) cylinder set measure $\mu_{t}$ is defined by

$$
\mu_{t}(C) \triangleq \frac{1}{(2 \pi t)^{-\frac{n}{2}}} \int_{E} e^{-\frac{|x|^{2}}{2 t}} \mathrm{~d} x
$$

where $C, P$ and $E$ are given above, $n=\operatorname{dim} P H$, and $\mathrm{d} x$ is the Lebesgue measure in $P H$. A measurable semi-norm on $H$ is a semi-norm $\|\cdot\|$ on $H$ with the property that for every $\epsilon>0$, there exists a finitedimensional projection $P_{0}$ such that for every finite-dimensional projection $P$ orthogonal to $P_{0}$ it holds 
that $\mu_{1}(\{x \in H:\|P x\|>\epsilon\})<\epsilon$. As a consequence of the definition of measurable semi-norm, every measurable semi-norm $\|\cdot\|$ is dominated by the Hilbert norm, i.e., there exists a constant $C$ such that $\|x\| \leqslant C|x|$ for all $x \in H$. If $\|\cdot\|$ is a measurable norm, we denote by $B$ the completion of $H$ with respect to $\|\cdot\|$. Then $B$ is a separable Banach space. There is a natural embedding $i$ from $H$ into $B$ whose image is dense in $B$. Then $i^{*}$ is also an embedding from $B^{*}$ into $H^{*}$. Since $H^{*}$ can be identified with $H$ we have the following inclusion relations $B^{*} \subset H \subset B$. Furthermore, we should note that an element $x$ of $H$ is in $B^{*}$ if and only if there exists a constant $C>0$ such that the inequality $|(x, y)| \leqslant C|| y \|$ holds for all $y$ in $H$. Then $\mu_{t}$ induces a cylinder set measure $m_{t}$ in $B$ as follows. If $y_{1}, \cdots, y_{n} \in B^{*}$ and $E$ is a Borel set of $\mathbb{R}^{n}$, we define

$$
m_{t}\left(\left\{x \in B:\left(\left\langle y_{1}, x\right\rangle, \cdots,\left\langle y_{n}, x\right\rangle\right) \in E\right\}\right) \triangleq \mu_{t}\left(\left\{x \in H:\left(\left(y_{1}, x\right), \cdots,\left(y_{n}, x\right)\right) \in E\right\}\right) .
$$

In [15], it was proved that $m_{t}$ is countably additive on the cylinder sets of $B$. By the Carathéodory extension theorem, it can be uniquely extended to the Borel sets of $B$ as a measure, denoted by $p_{t}$. The triple $(i, H, B)$ is called an abstract Wiener space and the measure $p_{t}$ is called the Wiener measure on $B$ with variance parameter $t$. For any $x \in B$ and Borel subset $A$ of $B$, we define $p_{t}(x, A) \triangleq p_{t}(A-x)$. By [14], one has the following formula for the Wiener measure.

Proposition 2.8. For any $s, t \in(0,+\infty)$ and $x, y \in B, p_{t}(x, \cdot)$ and $p_{s}(y, \cdot)$ are equivalent measures if and only if $s=t$ and $x-y \in H$. Otherwise they are mutually singular. Furthermore, it holds that $p_{t s}(A)=p_{t}\left(s^{-\frac{1}{2}} A\right)$ for any Borel subset $A$ of $B$.

In terms of the inclusion relations $B^{*} \subset H \subset B$, one has a useful product decomposition of Wiener measure $p_{t}$ as follows. Suppose $K$ is a finite-dimensional subspace of $B^{*}$ and $L$ is its annihilator in $B$. If $\left\{y_{1}, \cdots, y_{n}\right\}$ is an orthonormal basis of $K$ then we define $Q x=\sum_{j=1}^{n}\left\langle y_{j}, x\right\rangle y_{j}, x \in B$. Then $Q$ is a continuous linear operator from $B$ into $B$. Obviously, the range of $Q$ is $K$ and null space of $Q$ is $L$. We thus get $B=K \oplus L$. Let $K^{\perp}$ be the orthogonal complement of $K$ in $H$. Then $K^{\perp} \subset L$ and $L$ is the closure in $B$ of $K^{\perp}$. It is easy to check that the restriction of a measurable norm to a closed subspace is again a measurable norm. Thus there is a Wiener measure $p_{t}^{\prime}$ on the space $L$. Let $\mu_{t}^{\prime}$ denote the typical Gaussian measure in $K$ then in the Cartesian product decomposition $B=K \times L$ there holds $p_{t}=\mu_{t}^{\prime} \times p_{t}^{\prime}$.

The following exponential integrability of Wiener measure, discovered in [7], is very useful.

Theorem 2.9. (Fernique's Theorem) For any fixed $t \in(0, \infty)$, there exists $\epsilon=\epsilon\left(p_{t}\right)>0$ such that

$$
\int_{B} e^{\epsilon|| x \|^{2}} p_{t}(\mathrm{~d} x)<\infty .
$$

Denote by $\mathcal{B}(B)$ the collection of Borel sets of $B$. The following density result ([6, Proposition 39.8]) will also be used later.

Proposition 2.10. Suppose $\mu$ is a probability measure on $\mathcal{B}(B)$ so that for every $\varphi \in B^{*}$, there exists a constant $\epsilon=\epsilon\left(\|\varphi\|_{B^{*}}\right)>0$ such that $\int_{B} e^{\epsilon|\varphi|} \mathrm{d} \mu<\infty$ (Here, $|\varphi|$ stands for the absolute value of $\varphi$ ). Then $\mathcal{F} \triangleq\left\{P\left(\varphi_{1}, \cdots, \varphi_{n}\right): n \in \mathbb{N}, \varphi_{i} \in B^{*}, 1 \leqslant i \leqslant n, P\right.$ is a real polynomial with $n$ variables $\}$ is dense in $L^{p}(B, \mathcal{B}(B), \mu)$ for $1 \leqslant p<\infty$.

In what follows, $(i, H, B)$ is a given abstract Wiener space. For a real valued function $f$ defined on an open set $O$ of $B$, one has two definitions of derivatives. Firstly, for $x \in O$ if $f$ is $B$-Fréchet differentiable at $x$, we will denote the corresponding derivative at $x$ by $f^{\prime}(x)$. Secondly, we have a function defined by $g(h)=f(x+i h)$ which is a function on a neighborhood of 0 in $H$. If $g$ is $H$-Fréchet differentiable at 0 , we will denote the corresponding derivative at 0 by $D f(x)$. From [14] we see that the second derivative is weaker than the first derivative. The second derivative is sometimes called Malliavin derivative, which will be used in the proof of the Holmgren type theorem in this paper. For $n \geqslant 2$, we will use the notations $f^{n}(x)$ and $D^{n} f(x)$ to denote the corresponding higher order derivatives.

\subsection{A Divergence Theorem in Abstract Wiener Space}

In this subsection, we will give a brief exposition of surface measures and a divergence theorem in the abstract Wiener space $(i, H, B)$, developed in [13]. 
Firstly, we introduce the concepts of "smooth" functions and surfaces.

Definition 2.11. A real-valued function $g$ defined on an open subset $U$ of $B$ is called an $H$ - $C^{1}$ function if $g$ is continuous and $H$-Fréchet differentiable on $U$, the map $D g: U \rightarrow H^{*}$ is continuous and the vector $D g(x) \in B^{*}$ for each $x \in U$.

Definition 2.12. $\quad$ A subset $S$ (resp. $V$ ) of $B$ is called an $H-C^{1}$ surface (resp. to have an $H$ - $C^{1}$ boundary $\partial V$ ) if for each $x \in S$ (resp. $x \in \partial V$ ) there is an open neighborhood $U$ of $x$ in $B$ and an $H$ - $C^{1}$ function $g$ defined on $U$ such that $D g(x) \neq 0$ and $S \cap U=\{y \in U: g(y)=0\}$ (resp. $V \cap U=\{y \in U: g(y)<0\})$.

Secondly, we introduce the notion of local coordinate for the above $H-C^{1}$ surface $S$. We begin with the concept of normal projection:

Definition 2.13. A one dimensional orthogonal projection $P$ on $H$ is called a normal projection for $S$ at $x \in S$ if $|P(y-x)|=o(|y-x|)$ as $|y-x| \rightarrow 0$ for all $y \in S$ so that $y-x \in H$.

Denote by $I$ the identity operator on $H$. One can show the following result:

Proposition 2.14. $\quad$ There exists a unique map $N .: S \rightarrow \mathcal{L}\left(B ; B^{*}\right)$ such that

(1) For each $x \in S$ the restriction of $N_{x}$ to $H$ is a normal projection for $(S-x) \cap H$ at 0 ;

(2) For each $x$ the map $J_{x}=I-N_{x}$ is a homeomorphism of an open neighborhood of $x$ in $S$ onto an open subset in the null space of $N_{x}$;

(3) The map $N: S \rightarrow \mathcal{L}(H)$ is continuous.

For any $y \in S$, by Proposition 2.14, there is an element $h$ in $B^{*}$ with $|h|=1$ and a neighborhood $W$ of $y$ in $S$ such that $N_{y} h=h,\left|N_{w} h\right|>0$ for all $w$ in $W$, and $J_{y}=I-N_{y}$ is a homeomorphism of $W$ into the null space of $N_{y}$. We call the above element $h$ a unit normal vector at $y$, and $W$ a coordinate neighborhood of $y$. For a fixed $x$ in $B$ and $t>0$ we define a measure $\rho(t, x, W, \cdot)$ on the Borel sets of $W$ by

$$
\rho(t, x, W, E) \triangleq \frac{1}{\sqrt{2 \pi t}} \int_{J_{y}(E)} \frac{1}{\left|N_{J_{y}^{-1} z} h\right|} \exp \left[-\frac{\left|N_{y}\left(J_{y}^{-1} z-x\right)\right|^{2}}{2 t}\right] p_{t}^{\prime}\left(J_{y} x, \mathrm{~d} z\right)
$$

where $p_{t}^{\prime}$ is the Wiener measure on the null space of $N_{y}$. We call $\rho(t, x, W, \cdot)$ a local version of normal surface measure with dilation parameter $t$ and translation variable $x$.

One has the following result:

Theorem 2.15. For any $x \in B$ and $t>0$, there is a unique measure $\sigma_{t}(x, \cdot)$ on the Borel sets of $S$ such that for any local version of normal surface measure $\rho(t, x, W, \cdot)$ on a coordinate neighborhood $W$ in $S$ and any Borel subset $E$ of $W$, it holds that $\sigma_{t}(x, E)=\rho(t, x, W, E)$.

The measure $\sigma_{t}(x, \cdot)$ given in Theorem 2.15 is called a normal surface measure on $S$ with dilation parameter $t$ and translation variable $x$.

In the sequel, $V$ is a given nonempty open set of $B$ and has an $H-C^{1}$ boundary $\partial V$. We also need the following two concepts.

Definition 2.16. $\quad$ A map $\mathbf{n}: \partial V \rightarrow H^{*}$ is called an outward normal map for $V$ provided that $\mathbf{n}(y)$ is a unit normal vector at $y$ for the surface $\partial V$ and $y-s \mathbf{n}(y) \in V$ for any small $s>0$.

Definition 2.17. An ( $H$-valued) function $F$ defined on an open subset $U$ of $B$ is called to have finite divergence at $x \in U$ if $F$ is $H$-Fréchet differentiable at $x$ and $D F(x)$ is an operator of trace class on $H$. For such a function $F$, the divergence of $F$ at $x$ is defined by the trace of $D F(x)$, and denoted by $(\operatorname{div} F)(x)$.

The following result will play a key role in the proof of our Holmgren type theorem of infinite many variables.

Theorem 2.18. (Divergence Theorem) Assume that $F: V \cup \partial V \rightarrow H$ is a continuous function with finite divergence on $V$ and that $F$ is uniformly bounded with respect to the $B^{*}$-norm on $V$. If for some $x \in B$ and $t>0$, the function $|F(\cdot)|$ is $\sigma_{t}(x, \cdot)$-integrable on $\partial V$ and the trace class operator norm 
of $D F$ is $p_{t}(x, \cdot)$-integrable on $V$, then

$$
\int_{V}\left[(\operatorname{div} F)(y)-\frac{\langle F(y), y-x\rangle}{t}\right] p_{t}(x, \mathrm{~d} y)=\int_{\partial V}\langle F(y), \mathbf{n}(y)\rangle \sigma_{t}(x, \mathrm{~d} y) .
$$

\section{Cauchy-Kowalevski Type Theorem of Infinitely Many Variables}

This section is devoted to a study of the following form of initial value problem:

$$
\left\{\begin{array}{l}
\partial_{t}^{m} u(t, \mathbf{x})=f\left(t, \mathbf{x}, u, \partial_{\mathbf{x}}^{\beta} \partial_{t}^{j} u\right), \\
\left.\partial_{t}^{k} u(t, \mathbf{x})\right|_{t=0}=\phi_{k}(\mathbf{x}), \quad k=0,1, \cdots, m-1 .
\end{array}\right.
$$

Here $m$ is a given positive integer, $t \in \mathbb{R}, \mathbf{x}=\left(x_{i}\right) \in \mathbb{R}^{\infty}, \partial_{\mathbf{x}}^{\beta} \triangleq \partial_{x_{1}^{\beta_{1}}}^{\beta_{1}} \cdots \partial_{x_{n}^{\beta_{n}}}^{\beta_{n}}$ for $\beta=\left(\beta_{1}, \cdots, \beta_{n}, 0, \cdots\right) \in$ $\mathbb{N}^{(\mathbb{N})}$ (for some positive $n \in \mathbb{N}$ ), the unknown $u$ is a real-valued function depending on $t$ and $\mathbf{x} ; f$ is a nonlinear real-valued function depending on $t, \mathbf{x}, u$ and all of its derivatives of the form $\partial_{x}^{\beta} \partial_{t}^{j} u, \beta \in \mathbb{N}^{(\mathbb{N})}, j<$ $m, 1 \leqslant|\beta|+j \leqslant m$. Note that the values $u(0,(0)), \partial_{x}^{\beta} \partial_{t}^{j} u(0,(0)), \beta \in \mathbb{N}^{(\mathbb{N})}, j<m, 1 \leqslant|\beta|+j \leqslant m$ are determined by (3.1). For simplicity, we write these determined values by $u(0,(0))=u_{0}, \partial_{x}^{\beta} \partial_{t}^{j} u(0,(0))=$ $w_{\beta, j}^{0}, \beta \in \mathbb{N}^{(\mathbb{N})}, j<m, 1 \leqslant|\beta|+j \leqslant m$ and $\mathbf{w}^{0}=\left(w_{\beta, j}^{0}\right)_{\beta \in \mathbb{N}^{(\mathbb{N})}, j<m, 1 \leqslant|\beta|+j \leqslant m}$. One can see that $f$ is a function on $\mathbb{R} \times \mathbb{R}^{\infty} \times \mathbb{R} \times \mathbb{R}^{\infty}$ which is also a countable Cartesian product of $\mathbb{R}$. Therefore, we may identify $\mathbb{R} \times \mathbb{R}^{\infty} \times \mathbb{R} \times \mathbb{R}^{\infty}$ with $\mathbb{R}^{\infty}$. We suppose that $f$ is analytic near $\left(0,(0), u(0,(0)), \partial_{x}^{\beta} \partial_{t}^{j} u(0,(0))\right)$ with the monomial expansion $f\left(t,\left(x_{i}\right), u, \partial_{x}^{\beta} \partial_{t}^{j} u\right)=\sum_{\alpha \in \mathbb{N}^{(\mathbb{N})}} C_{\alpha}\left(t,\left(x_{i}\right), u-u_{0}, \partial_{x}^{\beta} \partial_{t}^{j} u-w_{\beta, j}^{0}\right)^{\alpha}$ and let $F(t, \mathbf{x}, u, \mathbf{w}) \triangleq \sum_{\alpha \in \mathbb{N}^{(N)}}\left|C_{\alpha}\right|(t, \mathbf{x}, u, \mathbf{w})^{\alpha}$, where $\mathbf{w}=\left(w_{\beta, j}\right)_{\beta \in \mathbb{N}^{(\mathbb{N})}, 1 \leqslant|\beta|+j \leqslant m, j<m} \in \mathbb{R}^{\infty}$ and $\mathbf{x}=\left(x_{i}\right) \in$ $\mathbb{R}^{\infty}$. We also suppose that for each $0 \leqslant k \leqslant m-1, \phi_{k}$ is analytic near $(0,(0))$ with monomial expansion $\phi_{k}(\mathbf{x})=\sum_{\alpha \in \mathbb{N}^{(\mathbb{N})}} C_{\alpha, k} \mathbf{x}^{\alpha}$ and let $\Phi_{k}(t, \mathbf{x}, u, \mathbf{w}) \triangleq \sum_{\alpha \in \mathbb{N}^{(\mathbb{N})}}\left|C_{\alpha, k}\right| \mathbf{x}^{\alpha}$.

By means of the majorant method, we can show the following Cauchy-Kowalevski type theorem of infinitely many variables:

Theorem 3.1. Suppose $1 \leqslant p<\infty$ and that the monomial expansions of $\Phi_{k}, 0 \leqslant k \leqslant m-1$ and $F$ near $(0,(0))$ are absolutely convergent at a point near $\infty$ in the topology $\mathcal{T}^{p}$. Then the Cauchy problem (3.1) admits a locally analytic solution (near (0)), which is unique in the class of analytic functions under the topology $\mathscr{T}_{p^{\prime}}$ where $p^{\prime}$ is the usual Hölder conjugate of $p$. Furthermore, the solution u is Fréchet differentiable with respect to $\ell^{p^{\prime}}$ in a neighborhood of (0) in the topology $\mathcal{T}^{p^{\prime}}$ and the corresponding Fréchet derivative $D u$ is continuous.

Example 3.2. (The Schrödinger operator of infinitely many number of particles) This example is from [1]. Suppose that $\left\{a_{k}\right\}_{k=1}^{\infty}$ is a sequence of nonnegative real numbers. The following operator

$$
(L u)(\mathbf{x}) \triangleq-\frac{1}{2} \sum_{k=1}^{\infty} a_{k}\left(\frac{\partial^{2} u(\mathbf{x})}{\partial x_{k}^{2}}-2 x_{k} \frac{\partial u(\mathbf{x})}{\partial x_{k}}\right), \quad \mathbf{x}=\left(x_{k}\right) \in \mathbb{R}^{\infty},
$$

for $u \in \mathcal{P}\left(\mathbb{R}^{\infty}\right)$ which is the space of cylindrical polynomials on $\mathbb{R}^{\infty}$, i.e., polynomials depending only on finitely many variables. Note that in quantum mechanics the operator $N_{k} \triangleq-\frac{1}{2} a_{k}\left(\frac{\partial^{2}}{\partial x_{k}^{2}}-2 x_{k} \frac{\partial}{\partial x_{k}}\right)$ is an operator of energy of one-dimensional harmonic oscillator with unit mass and frequency in the space of $L^{2}\left(\mathbb{R}, \frac{e^{-x_{k}^{2}}}{\sqrt{\pi}} \mathrm{d} x_{k}\right)$. Thus the operator (3.2) describes a system consisting infinitely many noninteracting oscillators with frequency $a_{k} \geqslant 0, k \in \mathbb{N}$. If $a_{1}=1$ and we let $t=x_{1}$ then the equation $L u=0$ is equivalent to the following one:

$$
\frac{\partial^{2} u(t, \mathbf{x})}{\partial t^{2}}=2 t \frac{\partial u(t, \mathbf{x})}{\partial t}-\sum_{k=2}^{\infty} a_{k}\left(\frac{\partial^{2} u(t, \mathbf{x})}{\partial x_{k}^{2}}-2 x_{k} \frac{\partial u(t, \mathbf{x})}{\partial x_{k}}\right)
$$

which is of form (3.1). One can easily see that the above equation satisfies the assumption in Theorem 3.1 if and only if there exist $\left(b_{k}\right),\left(c_{k}\right) \in \mathbb{R}^{\infty}$ such that $\sum_{k=2}^{\infty}\left(\frac{1}{\left|b_{k}\right|^{p}}+\frac{1}{\left|c_{k}\right|^{p}}\right)<\infty$ and $\sum_{k=2}^{\infty}\left(\left|a_{k} b_{k}\right|+2\left|a_{k} c_{k}\right|\right)<\infty$. 
For example, if $p=2$ and let $a_{k}=\frac{1}{k^{3}}, b_{k}=c_{k}=\frac{1}{k}, k=2,3, \cdots$. Then $\sum_{k=2}^{\infty}\left(\left|a_{k} b_{k}\right|+2\left|a_{k} c_{k}\right|\right)=$ $\sum_{k=2}^{\infty} \frac{3}{k^{2}}<\infty$.

One can find similar examples such as the Hamilton-Jacobi equation in infinite dimensions ([3]) and the Laplacian defined on $\ell^{2}$ by Malliavin derivatives ([14]).

Now, let us consider the Cauchy problem of the following first order linear homogenous partial differential equation of infinitely many variables:

$$
\left\{\begin{array}{l}
\partial_{t} u(t, \mathbf{x})-\sum_{i=1}^{\infty} a_{i}(t, \mathbf{x}) \partial_{x_{i}} u(t, \mathbf{x})-b(t, \mathbf{x}) u(t, \mathbf{x})=0 \\
\left.u(t, \mathbf{x})\right|_{t=0}=\phi(\mathbf{x})
\end{array}\right.
$$

Here $t \in \mathbb{R}, \mathbf{x}=\left(x_{i}\right)_{i=1}^{\infty} \in \mathbb{R}^{\infty}$, the unknown $u$ is a real-valued function depending on $t$ and $\mathbf{x}$, and the data $a_{i}(t, \mathbf{x})$ 's, $b(t, \mathbf{x})$ and $\phi$ are analytic near $(0)$. Let

$$
G(t, \mathbf{x}, \mathbf{w}) \triangleq \sum_{i=1}^{\infty} a_{i}(t, \mathbf{x}) w_{i}+w_{0} b(t, \mathbf{x}), \quad \mathbf{w}=\left(w_{j}\right)_{j=0}^{\infty} \in \mathbb{R}^{\infty}
$$

By modifying the proof of Theorem 3.1, we can show the following result.

Corollary 3.3. Suppose $1 \leqslant p<\infty$, the monomial expansion of $G$ near $(0)$ is absolutely convergent at a point near $\infty$ in the topology $\mathcal{T}^{p}$, and $D_{\phi}^{(0)}=\mathbb{R}^{\infty}$. Then there exists $r \in(0, \infty)$, independent of $\phi$, such that the equation (3.3) admits locally an analytic solution u near (0) and $D_{u}^{(0)} \supset B_{r}^{p^{\prime}}$. Furthermore, $u$ is Fréchet differentiable with respect to $\ell^{p^{\prime}}$ in $B_{r}^{p^{\prime}}$, and the corresponding Fréchet derivative Du is continuous.

\section{Holmgren Type Theorem of Infinitely Many Variables}

Denote by $\Xi$ the set of real-valued functions, defined locally near $(0)$ in $\mathbb{R}^{\infty}$, which are Fréchet differentiable with respect to $\ell^{2}$ and whose Fréchet derivative are locally continuous near $(0)$ in the $\mathscr{T}_{2}$ topology.

In this section, we shall establish the following Holmgren type theorem of infinitely many variables.

Theorem 4.1. Suppose the monomial expansion of $G$ (defined by (3.4)) near (0) is absolutely convergent at a point near $\infty$ in the topology $\mathcal{T}^{\frac{1}{2}}$ and $\phi \in \Xi$. Then the solution to (3.3) is locally unique in the class $\Xi$.

In the rest, we shall give a sketch of the proof of Theorem 4.1.

It suffices to show that $U \in \Xi$ must be 0 provided that $U$ solves $(3.3)$ with $\phi(\cdot)=0$. Without loss of generality, we assume that the data $a_{i}(t, \mathbf{x}) \equiv a_{i}(\mathbf{x})(i=1,2, \cdots)$ and $b(t, \mathbf{x}) \equiv b(\mathbf{x})$, i.e., they are independent of $t$. By our assumption, for each $i \in \mathbb{N}, a_{i}(\mathbf{x})$ (resp. $b(\mathbf{x})$ ) has a monomial expansion near (0): $a_{i}(\mathbf{x})=\sum_{\alpha \in \mathbb{N}(\mathbb{N})} a_{i, \alpha} \mathbf{x}^{\alpha}\left(\right.$ resp. $\left.b(\mathbf{x})=\sum_{\alpha \in \mathbb{N}^{(\mathbb{N})}} a_{0, \alpha} \mathbf{x}^{\alpha}\right)$. Then, there exist $\rho_{0}>0$ and $0<s_{j}, t_{i}<1,0 \leqslant i<\infty, 1 \leqslant j<\infty$ such that $\sum_{j=1}^{\infty} s_{j}^{\frac{1}{2}}+\sum_{i=0}^{\infty} t_{i}^{\frac{1}{2}}=1$ and

$$
\sum_{i=0}^{\infty}\left[\sum_{\alpha \in \mathbb{N}(\mathbb{N})}\left|a_{i, \alpha}\right|\left(\frac{\rho_{0}}{s_{j}}\right)^{\alpha}\right] \frac{\rho_{0}}{t_{i}}<\infty
$$

Now we need to introduce a suitable transformation of variables. In a neighborhood of $(0)$ in $\mathscr{T}_{2}$, put

$$
t^{\prime} \triangleq t+\sum_{i=1}^{\infty} x_{i}^{2}, \quad x_{i}^{\prime} \triangleq x_{i}, i=1,2, \cdots, \quad \widetilde{U}\left(t^{\prime}, x_{1}^{\prime}, \cdots, x_{i}^{\prime}, \cdots\right) \triangleq U\left(t^{\prime}-\sum_{i=1}^{\infty}\left(x_{i}^{\prime}\right)^{2}, x_{1}^{\prime}, \cdots, x_{i}^{\prime}, \cdots\right) .
$$

Then $\widetilde{U} \in \Xi$, and

$$
\left(1-2 \sum_{i=1}^{\infty} x_{i}^{\prime} a_{i}\left(\mathbf{x}^{\prime}\right)\right) \partial_{t^{\prime}} \widetilde{U}=\sum_{i=1}^{\infty} a_{i}\left(\mathbf{x}^{\prime}\right) \partial_{x_{i}^{\prime}} \widetilde{U}+b\left(\mathbf{x}^{\prime}\right) \widetilde{U}
$$


where $\mathbf{x}^{\prime}=\left(x_{i}^{\prime}\right) \in \ell^{2}$. Clearly, $\left|2 \sum_{i=1}^{\infty} x_{i}^{\prime} a_{i}\left(\mathbf{x}^{\prime}\right)\right|<1$ in a neighborhood of (0) in $\mathcal{T}_{2}$. Thus (4.2) can be written as $\partial_{t^{\prime}} \widetilde{U}=\sum_{i=1}^{\infty} \widetilde{a}_{i}\left(\mathbf{x}^{\prime}\right) \partial_{x_{i}^{\prime}} \widetilde{U}+\widetilde{b}\left(\mathbf{x}^{\prime}\right) \widetilde{U}$, where $\widetilde{a}_{i}\left(\mathbf{x}^{\prime}\right)=\frac{a_{i}\left(\mathbf{x}^{\prime}\right)}{1-2 \sum_{i=1}^{\infty} x_{i}^{\prime} a_{i}\left(\mathbf{x}^{\prime}\right)}, 1 \leqslant i<\infty$ and $\widetilde{b}\left(\mathbf{x}^{\prime}\right)=\frac{b\left(\mathbf{x}^{\prime}\right)}{1-2 \sum_{i=1}^{\infty} x_{i}^{\prime} a_{i}\left(\mathbf{x}^{\prime}\right)}$. Now let $A_{0}=t_{0}^{\frac{1}{4}}, A_{i}=\max \left\{t_{i}^{\frac{1}{4}}, s_{i}^{\frac{1}{4}}\right\}, 1 \leqslant i<\infty$. Then, $\sum_{i=0}^{\infty} A_{i}^{2}<\sum_{j=1}^{\infty} s_{j}^{\frac{1}{2}}+$ $\sum_{i=0}^{\infty} t_{i}^{\frac{1}{2}}=1$ and by $(4.1)$ there exists some $\rho_{1} \in\left(0, \rho_{0}\right)$ such that $\sum_{i=0}^{\infty}\left[\sum_{\alpha \in \mathbb{N}(\mathbb{N})}\left|a_{i, \alpha}\right|\left(\frac{\rho_{1}}{A_{j}^{4}}\right)_{j \in \mathbb{N}}^{\alpha}\right] \frac{\rho_{1}}{A_{i}^{4}}<$ $\frac{1}{2}$. Write the monomial expansions of $\widetilde{b}\left(\mathbf{x}^{\prime}\right)$ and $\widetilde{a}_{i}\left(\mathbf{x}^{\prime}\right), 1 \leqslant i<\infty$ near $(0)$ respectively as $\widetilde{b}\left(\mathbf{x}^{\prime}\right)=$ $\sum_{\alpha \in \mathbb{N}(\mathbb{N})} a_{0, \alpha}^{\prime} \mathbf{x}^{\prime \alpha}$ and $\widetilde{a}_{i}\left(\mathbf{x}^{\prime}\right)=\sum_{\alpha \in \mathbb{N}(\mathbb{N})} a_{i, \alpha}^{\prime} \mathbf{x}^{\prime \alpha}, 1 \leqslant i<\infty$. It is easily seen that

$$
\sum_{i=0}^{\infty}\left[\sum_{\alpha \in \mathbb{N}^{(\mathbb{N})}}\left|a_{i, \alpha}^{\prime}\right|\left(\frac{\rho_{1}}{A_{j}^{4}}\right)_{j \in \mathbb{N}}^{\alpha}\right] \frac{\rho_{1}}{A_{i}^{4}}<\infty .
$$

Formally write

$$
\begin{aligned}
& G_{1}[\widetilde{U}] \triangleq \partial_{t^{\prime}} \widetilde{U}-\sum_{i=1}^{\infty} \widetilde{a}_{i}\left(\mathbf{x}^{\prime}\right) \partial_{x_{i}^{\prime}} \widetilde{U}-\widetilde{b}\left(\mathbf{x}^{\prime}\right) \widetilde{U} \\
& G_{2}[W] \triangleq-\partial_{t^{\prime}} W+\sum_{i=1}^{\infty} \partial_{x_{i}^{\prime}}\left[\widetilde{a}_{i}\left(\mathbf{x}^{\prime}\right) W\right]+\left[-\widetilde{b}\left(\mathbf{x}^{\prime}\right)+\frac{t^{\prime}}{A_{0}^{3}}-\sum_{i=1}^{\infty} \frac{x_{i}^{\prime} \widetilde{a}_{i}\left(\mathbf{x}^{\prime}\right)}{A_{i}^{3}}\right] W
\end{aligned}
$$

where the function $W$ will be defined later. For a sufficient small positive number $\lambda$, we put

$$
H_{\lambda}=\left\{\left(t^{\prime}, x_{1}^{\prime}, \cdots, x_{i}^{\prime}, \cdots\right) \in \mathbb{R}^{\infty}: \sum_{i=1}^{\infty}\left(x_{i}^{\prime}\right)^{2}<t^{\prime}<\lambda\right\}
$$

Also, denote the boundary of $H_{\lambda}$ by $l_{\lambda} \cup I_{\lambda} \cup k_{\lambda}$ where

$$
\begin{aligned}
& l_{\lambda}=\left\{\left(t^{\prime}, x_{1}^{\prime}, \cdots, x_{i}^{\prime}, \cdots\right) \in \mathbb{R}^{\infty}: t^{\prime}=\lambda, \sum_{i=1}^{\infty}\left(x_{i}^{\prime}\right)^{2}<\lambda\right\}, \\
& I_{\lambda}=\left\{\left(t^{\prime}, x_{1}^{\prime}, \cdots, x_{i}^{\prime}, \cdots\right) \in \mathbb{R}^{\infty}: t^{\prime}=\lambda, \sum_{i=1}^{\infty}\left(x_{i}^{\prime}\right)^{2}=t^{\prime}\right\}, \\
& k_{\lambda}=\left\{\left(t^{\prime}, x_{1}^{\prime}, \cdots, x_{i}^{\prime}, \cdots\right) \in \mathbb{R}^{\infty}: t^{\prime}<\lambda, \sum_{i=1}^{\infty}\left(x_{i}^{\prime}\right)^{2}=t^{\prime}\right\} .
\end{aligned}
$$

From the definition one can see that $H_{\lambda}$ is an open subset of $\ell^{2}$ and $\left.\widetilde{U}\right|_{I_{\lambda} \cup k_{\lambda}}=0$. Let

$$
F\left(t^{\prime}, \mathbf{x}^{\prime}\right) \triangleq\left(\frac{t^{\prime}}{A_{0}},-\frac{\widetilde{a}_{1}\left(\mathbf{x}^{\prime}\right)}{A_{1}}, \cdots,-\frac{\widetilde{a}_{i}\left(\mathbf{x}^{\prime}\right)}{A_{i}}, \cdots\right), \quad t^{\prime} \in \mathbb{R}, \mathbf{x}^{\prime}=\left(x_{i}^{\prime}\right) \in \ell^{2},
$$

$H \triangleq\left\{\left(t^{\prime}, x_{1}^{\prime}, \cdots, x_{i}^{\prime}, \cdots\right) \in \mathbb{R}^{\infty}:\left(\frac{t^{\prime}}{A_{0}}\right)^{2}+\sum_{i=1}^{\infty}\left(\frac{x_{i}^{\prime}}{A_{i}}\right)^{2}<\infty\right\}$ and $B \triangleq\left\{\left(t^{\prime}, x_{1}^{\prime}, \cdots, x_{i}^{\prime}, \cdots\right) \in \mathbb{R}^{\infty}:\left(t^{\prime}\right)^{2}+\right.$ $\left.\sum_{i=1}^{\infty}\left(x_{i}^{\prime}\right)^{2}<\infty\right\}$. We can view $B$ as $\mathbb{R} \times \ell^{2}$ and we will use this convention later. Since $\sum_{i=0}^{\infty} A_{i}^{2}<\infty$, we have the natural inclusion map $i$ which maps $H$ into $B$ such that the triple $(i, H, B)$ is an abstract Wiener space. Since $H=H^{*}, i^{*} B^{*}$ can be characterized by a subset of $H$ which will be denoted by $B^{*}$. Precisely, $B^{*} \triangleq\left\{h \in H\right.$ : there exists $C_{h} \in(0,+\infty)$ such that $\left|(h, g)_{H}\right| \leqslant C_{h}|| i g \|_{B}$ for any $\left.g \in H\right\}$. A simple computation shows that $B^{*}=\left\{\left(t^{\prime}, x_{1}^{\prime}, \cdots, x_{i}^{\prime}, \cdots\right): \frac{\left(t^{\prime}\right)^{2}}{A_{0}^{4}}+\sum_{i=1}^{\infty} \frac{\left(x_{i}^{\prime}\right)^{2}}{A_{i}^{4}}<\infty\right\}$. In order to apply Theorem 2.18, we need the following result:

Proposition 4.2. For a sufficiently small $\lambda>0$ it holds that

(a) $F$ is uniformly bounded in $B^{*}$ norm, i.e., $\sup _{\left(t^{\prime}, x^{\prime}\right) \in H_{\lambda}} \sum_{i=1}^{\infty}\left[\frac{\widetilde{a}_{i}\left(x^{\prime}\right)}{A_{i}^{3}}\right]^{2}<\infty$;

(b) $F$ maps $H_{\lambda} \cup l_{\lambda} \cup k_{\lambda} \cup I_{\lambda}$ into a bounded subset of $H$, i.e., $\sup _{\left(t^{\prime}, x^{\prime}\right) \in H_{\lambda} \cup l_{\lambda} \cup k_{\lambda} \cup I_{\lambda}} \sum_{i=1}^{\infty}\left[\frac{\widetilde{a}_{i}\left(x^{\prime}\right)}{A_{i}^{2}}\right]^{2}<\infty$;

(c) $F$ is $H$-Fréchet differentiable with $D F$ being a trace class operator and the trace norm $\|D F\|_{1}$ is $p$ integrable on $H_{\lambda}$. 
It is easy to see that both $l_{\lambda}$ and $k_{\lambda}$ are two differentiable surfaces in $B$, by [13, Remark 2], both $l_{\lambda}$ and $k_{\lambda}$ are two $H-C^{1}$ surfaces in the abstract Wiener space $(i, H, B)$. Denote by $p($ resp. $\sigma)$ the corresponding Wiener measure on $B$ (resp. normal surface measure given in Theorem 2.15) with parameters $x=0$ and $t=1$. We may show the following result (which means that, for any $\lambda>0, \sigma$ is a finite measure):

Lemma 4.3. For any $\lambda>0$, it holds that $\sigma\left(l_{\lambda} \cup k_{\lambda} \cup I_{\lambda}\right)<\infty$.

By Lemma 4.3 and the second conclusion in Proposition 4.2, it follows that $|F|_{H}$ is $\sigma$-integrable on $l_{\lambda} \cup k_{\lambda} \cup I_{\lambda}$.

Note that the Divergence Theorem, i.e., Theorem 2.18 requires the boundary to be a "smooth" surface but the boundary of $H_{\lambda}$ is union of two "smooth" surfaces. However, similar arguments in [13] can be modified to show that the Divergence Theorem also holds in this following case:

Theorem 4.4. Assume that $\mathcal{F}: H_{\lambda} \cup l_{\lambda} \cup k_{\lambda} \cup I_{\lambda} \rightarrow H$ is a continuous function with finite divergence on $H_{\lambda}$ and that $\mathcal{F}$ is uniformly bounded with respect to the $B^{*}$-norm on $H_{\lambda}$. If the function $|\mathcal{F}(\cdot)|$ is integrable with respect to the normal surface measure $\sigma(\cdot)$ on $l_{\lambda} \cup k_{\lambda} \cup I_{\lambda}$, and the trace class norm of $D \mathcal{F}$ is p-integrable on $H_{\lambda}$, then

$$
\int_{H_{\lambda}}[(\operatorname{div} \mathcal{F})(y)-\langle\mathcal{F}(y), y\rangle] p(\mathrm{~d} y)=\int_{l_{\lambda} \cup k_{\lambda} \cup I_{\lambda}}\langle\mathcal{F}(y), \mathbf{n}(y)\rangle \sigma(\mathrm{d} y) .
$$

By Proposition 4.2 and Lemma 4.3, we conclude that $H_{\lambda}$ and the function $F$ defined by (4.4) satisfies the assumptions in Theorem 4.4. If $W$ is Frèchet differentiable respect to $B$ with continuous Frèchet derivatives, then $F_{0}=W \widetilde{U} F$ satisfies the assumptions in Theorem 4.4. Clearly, $\operatorname{div}(W \widetilde{U} F)=A_{0} \partial_{t^{\prime}}\left[\frac{W \widetilde{U}}{A_{0}}\right]-$ $\sum_{i=1}^{\infty} A_{i} \partial_{x_{i}^{\prime}}\left[\frac{\widetilde{a}_{i} W \widetilde{U}}{A_{i}}\right]$ and $\langle W \widetilde{U} F(y), y\rangle=\frac{W \widetilde{U} \cdot t^{\prime}}{A_{0}^{3}}-\sum_{i=1}^{\infty} \frac{\widetilde{a}_{i}\left(\mathbf{x}^{\prime}\right) W \widetilde{U} \cdot x_{i}^{\prime}}{A_{i}^{3}}$, where $y=\left(t^{\prime}, \mathbf{x}^{\prime}\right)=\left(t^{\prime},\left(x_{i}^{\prime}\right)_{i=1}^{\infty}\right) \in H$. Apply Theorem 4.4, we have

$$
\int_{H_{\lambda}}\left(W G_{1}[\widetilde{U}]-\widetilde{U} G_{2}[W]\right) p(\mathrm{~d} y)=\frac{\lambda}{A_{0}^{2}} \int_{l_{\lambda}} W \widetilde{U} \sigma(\mathrm{d} y) .
$$

Let $b^{\prime}\left(\mathbf{x}^{\prime}\right) \triangleq\left[\sum_{i=1}^{\infty} \partial_{x_{i}^{\prime}} \widetilde{a}_{i}\left(\mathbf{x}^{\prime}\right)-\widetilde{b}\left(\mathbf{x}^{\prime}\right)+\frac{t^{\prime}}{A_{0}^{3}}-\sum_{i=1}^{\infty} \frac{x_{i}^{\prime} \tilde{a}_{i}\left(\mathbf{x}^{\prime}\right)}{A_{i}^{3}}\right]$. Then $G_{2}[W]=-\partial_{t^{\prime}} W+\sum_{i=1}^{\infty} \widetilde{a}_{i}\left(\mathbf{x}^{\prime}\right) \partial_{x_{i}^{\prime}} W+$ $b^{\prime}\left(\mathbf{x}^{\prime}\right) W$. Let $G^{\prime}\left(\mathbf{x}^{\prime}, \mathbf{w}\right) \triangleq \sum_{i=1}^{\infty} \widetilde{a}_{i}\left(\mathbf{x}^{\prime}\right) w_{i}+w_{0} b\left(\mathbf{x}^{\prime}\right)$, where $\mathbf{w}=\left(w_{j}\right)_{j=0}^{\infty} \in \mathbb{R}^{\infty}$ and $\mathbf{x}=\left(x_{i}\right)_{i=1}^{\infty} \in \mathbb{R}^{\infty}$. Recall that $\sum_{i=0}^{\infty}\left[\sum_{\alpha \in \mathbb{N}^{(\mathbb{N})}}\left|a_{i, \alpha}^{\prime}\right|\left(\frac{\rho_{1}}{A_{j}^{4}}\right)^{\alpha}\right] \frac{\rho_{1}}{A_{i}^{4}}<\infty$. Hence, for any $\rho \in\left(0, \rho_{1}\right)$, the monomial expansion of $G^{\prime}$ is absolutely convergent at $\left(\left(\frac{\rho}{A_{j}}\right)_{j=0}^{\infty},\left(\frac{\rho}{A_{i}}\right)_{i=1}^{\infty}\right)$, which is a point near $\infty$ in the topology $\mathcal{T}^{2}$ by the fact that $\sum_{i=0}^{\infty} A_{i}^{2}<1$. Therefore, the equation $G_{2}[W]=0$ satisfies the assumptions in Corollary 3.3. Hence, for any $n \geqslant 0$ and $k_{1}, \cdots, k_{n} \in \mathbb{N}$, there is an analytic solution $W$ to $G_{2}[W]=0$ and $\left.W\right|_{t^{\prime}=\lambda}=\left(x_{1}^{\prime}\right)^{k_{1}} \cdots\left(x_{n}^{\prime}\right)^{k_{n}}$. From Corollary 3.3, it follows that the monomial expansion of $W$ is absolutely convergent in a neighborhood of (0) containing $H_{\lambda}$ for sufficiently small $\lambda$ and $W$ is Frèchet differentiable with respect to $B$ and the Frèchet derivative is continuous in this neighborhood. Therefore, for any sufficiently small $\lambda>0$, applying (4.5), we arrive at

$$
\int_{l_{\lambda}}\left(x_{1}^{\prime}\right)^{k_{1}} \cdots\left(x_{n}^{\prime}\right)^{k_{n}} \widetilde{U} \sigma(\mathrm{d} y)=0,
$$

for any $n \geqslant 0$ and $k_{1}, \cdots, k_{n} \in \mathbb{N}$. Let $L_{\lambda}=\left\{\left(t^{\prime}, x_{1}^{\prime}, \cdots, x_{i}^{\prime}, \cdots\right) \in \mathbb{R}^{\infty}: t^{\prime}=\lambda, \sum_{i=1}^{\infty}\left(x_{i}^{\prime}\right)^{2}<\infty\right\}$, then the surface measure $\sigma^{\prime}$ on $L_{\lambda}$ is identified with the Gaussian measure with parameters $x=0$ and $t=1$ on the Hilbert space $H_{0}=\left\{\left(x_{1}^{\prime}, \cdots, x_{i}^{\prime}, \cdots\right) \in \mathbb{R}^{\infty}: \sum_{i=1}^{\infty}\left(x_{i}^{\prime}\right)^{2}<\infty\right\}$. Note that (4.6) is equivalent to

$$
\int_{L_{\lambda}}\left(x_{1}^{\prime}\right)^{k_{1}} \cdots\left(x_{n}^{\prime}\right)^{k_{n}} \chi_{l_{\lambda}} \widetilde{U} \sigma^{\prime}(\mathrm{d} y)=0 .
$$

We also need the following density result.

Lemma 4.5. $\operatorname{span}\left\{\left(x_{1}^{\prime}\right)^{k_{1}} \cdots\left(x_{n}^{\prime}\right)^{k_{n}}: n \geqslant 0, k_{1}, \cdots, k_{n} \in \mathbb{N}\right\}$ is dense in $L^{2}\left(L_{\lambda}, \sigma^{\prime}\right)$. 
One may check that $L^{2}\left(l_{\lambda}, \sigma\right)=\chi_{l_{\lambda}} \cdot L^{2}\left(L_{\lambda}, \sigma^{\prime}\right)$. From Lemma 4.5 and noting the continuity of $\widetilde{U}$, we deduce that $\widetilde{U} \equiv 0$ on $l_{\lambda}$ for any sufficiently small $\lambda>0$ and hence $\left.\widetilde{U}\right|_{k_{\lambda}}=0$. Therefore, $\widetilde{U} \equiv 0$ on $H_{\lambda}$ for any sufficiently small $\lambda>0$. This implies that $U \equiv 0$ at a neighborhood of (0) in the $\mathcal{T}_{2}$ topology restricted on the half space $t>0$. By the same way we can prove that $U \equiv 0$ at a neighborhood of (0) the $\mathcal{T}_{2}$ topology restricted on the half space $t<0$. Finally, by the continuity of $U$ we have $U \equiv 0$ at a neighborhood of (0) in the $\mathcal{T}_{2}$ topology.

Acknowledgements The research of the first author is supported by NSFC under grant 11501384; The research of the second author is supported by NSFC under grant 11221101, the NSFC-CNRS Joint Research Project under grant 11711530142 and the PCSIRT under grant IRT_16R53 from the Chinese Education Ministry.

\section{References}

1 Berezanskiı Y. Selfadjoint operators in spaces of functions of infinitely many variables. Translations of Mathematical Monographs, American Mathematical Society, Providence, RI, 1986

2 Chaperon M. On the Cauchy-Kowalevski theorem. Enseign. Math. 2009, 55: 359-371

3 Crandall M,Lions P. Hamilton-Jacobi equations in infinite dimensions. I. Uniqueness of viscosity solutions. J. Funct. Anal. 1985, 62: 379-396

4 Defant A, Maestre M, Prengel C. Domains of convergence for monomial expansions of holomorphic functions in infinitely many variables. J. Reine Angew. Math. 2009, 634: 13-49

5 Dineen S. Complex analysis on infinite-dimensional spaces. Springer Monographs in Mathematics, Springer-Verlag, London, 1999

6 Driver B. Probability Tools with Examples. http://www.math.ucsd.edu/ bdriver/280_1314webs/280_lecture_notes.htm.

7 Fernique X. Intégrabilité des vecteurs gaussiens. C. R. Acad. Sci. Paris Sér. A. 1970, 270: 1698-1699

8 Fréchet M. Une définition fontionnelle des polynômes. Nouv. Ann. Math. 1909, 9: 145-130

9 Fréchet M. Sur les fonctionnelles continues. Ann. Sci. Ecole Norm. Sup. 1910, 27: 193-216

10 Friedman A. A new proof and generalizations of the Cauchy-Kowalewski theorem. Trans. Amer. Math. Soc. 1961, 98: $1-20$

11 Gâteaux R. Sur les fonctionnelles continues et les fonctionnelles analytiques. C. R. Acad. Sci. Paris, Ser. A. 1913, 157: $325-327$

12 Gâteaux R. Fonctions d'une infinité des variables indépendantes. Bull. Soc. Math. France. 1919, 47: 70-96

13 Goodman V. A divergence theorem for Hilbert space. Trans. Amer. Math. Soc. 1972, 164: 411-426

14 Gross L. Potential theory on Hilbert space. J. Funct. Anal. 1967, 1: 123-181

15 Gross L. Abstract Wiener spaces. Proc. Fifth Berkeley Sympos. Math. Statist. and Probability, Vol. II: Contributions to Probability Theory, Univ. California Press, Berkeley, Calif., 1967, Part 1: 31-42

16 Hedenmalm H. On the uniqueness theorem of Holmgren. Math. Z. 2015, 281: 357-378

17 Hilbert D. Wesen und Ziele einer Analysis der unendlichvielen unabhängigen Variablen. Rend. Circ. Mat. Palermo. 1909, 27: 59-74

18 Holmgren E Über Systeme von linearen partiellen Differentialgleichungen. Öfversigt af Kongl. Vetenskaps-Academien Förhandlinger. 1901, 58: 91-103

19 Hörmander L. A remark on Holmgren's uniqueness theorem. J. Differential Geom. 1971/72, 6: 129-134

20 Hörmander L. A uniqueness theorem for second order hyperbolic differential equations. Comm. Partial Differential Equations. 1992, 17: 699-714

21 John F. On linear partial differential equations with analytic coefficients. Unique continuation of data. Comm. Pure Appl. Math. 1949, 2: 209-253

22 von Koch h. Sur les système d'ordie infinite d'equations différentielles. Öfversigt af Kongl. Svenska VetenskapsAkademiens Forhandlinger. 1899, 61: 395-411

23 Lascar B. Théorème de Cauchy-Kovalewsky et thorème d'unicité d'Holmgren pour des fonctions d'une infinité de variables. C. R. Acad. Sci. Paris Sér. A-B. 1976, 282: A691-A694

24 Lascar B. Théorème de Cauchy-Kovalevsky et thorème d'unicité d'Holmgren pour des fonctions analytiques d'une infinité de variables. North-Holland Mathematics Studies, Advances in holomorphy, North-Holland Publishing Co., Amsterdam-New York, 1979, 485-508

25 Lempert L. The Dolbeault complex in infinite dimensions. II. J. Amer. Math. Soc. 1999, 12: 775-793

26 Mogilevskaja L. The analyticity of the solutions of differential equations in a Banach space. Dokl. Akad. Nauk SSSR. 1976, 228: 30-33

27 Nirenberg L. An abstract form of the nonlinear Cauchy-Kowalewski theorem. J. Differential Geom. 1972, 6: 561-576 
28 Nishida T. A note on a theorem of Nirenberg. J. Differential Geom. 1977, 12: 629-633

29 Ovsjannikov L. Singular operator in the scale of Banach spaces. Dokl. Akad. Nauk SSSR. 1965, 163: 819-822

30 Ryan R. Holomorphic mappings on $\ell^{1}$. Trans. Amer. Math. Soc. 1987, 302: 797-811

31 Safonov M. The abstract Cauchy-Kovalevskaya theorem in a weighted Banach space. Comm. Pure Appl. Math. 1995, 48: 629-637

32 Trèves F. An abstract nonlinear Cauchy-Kovalevska theorem. Trans. Amer. Math. Soc. 1970, 150: 77-92

33 Yamanaka T. A Cauchy-Kovalevskaja type theorem in the Gevrey class with a vector-valued time variable. Comm. Partial Differential Equations. 1992, 17: 1457-1502

34 Yu J Y, Zhang X. Cauchy-Kowalevski and Holmgren type theorems with infinite number of variables. Preprint

35 Zubelevich O. On the majorant method in the Cauchy-Kovalevskaya problem. Math. Notes. 2001, 69: 329-339 